\title{
Systematic studies of di-jet imbalance measurements in STAR
}

\author{
Nick Elsey for the STAR Collaboration* \\ Wayne State University \\ E-mail: dx5412@wayne.edu
}

\begin{abstract}
STAR has previously reported in $\mathrm{Au}+\mathrm{Au}$ collisions at $\sqrt{s_{N N}}=200 \mathrm{GeV}$ significant transverse momentum imbalance of a specific set of di-jets selected with "hard cores", i.e. with a constituent transverse momentum cut of $2 \mathrm{GeV} / c$. After reclustering these same di-jets with a lower constituent cut of $200 \mathrm{MeV} / c$, the di-jet balance is restored to the level of $\mathrm{p}+\mathrm{p}$ collisions within the original cone size of $R=0.4$. The interpretation of these results as resulting from tangential bias with restricted in-medium path lengths promises Jet Geometry Engineering of jet production vertices through systematic variations of parameters such as centrality, the constituent $p_{T}$ cutoff, and the initial imbalance between the hard cores. In these proceedings, we present a systematic exploration of jet quenching effects on di-jets sampled from a larger parameter space and explore in detail the possibilities and limitations of using these different di-jet definitions to constrain the influence of geometry on partonic energy loss in the quark gluon plasma created in heavy-ion collisions.
\end{abstract}

International Conference on Hard and Electromagnetic Probes of High-Energy Nuclear Collisions 30 September - 5 October 2018

Aix-Les-Bains, Savoie, France

${ }^{*}$ Speaker. 


\section{Introduction}

The properties of the quark-gluon plasma (QGP) formed in relativistic heavy-ion collisions can be studied using highly energetic partons created in hard scatterings in the initial stages of the collisions. These high-energy partons lose energy as they propagate through the QGP and fragment, before hadronizing into a collimated spray of particles called a jet. Measurements of the energy lost due to interactions with the medium (partonic energy loss, also known as jet quenching) can be used to infer properties of both the QGP and the jet itself. By comparing measurements in heavy-ion collisions to similar measurements done in proton+proton $(p+p)$ collisions (which are well described by pQCD at RHIC energies [1]), the effects of jet quenching can be extracted.

RHIC at Brookhaven National Laboratory has collided gold nuclei at $\sqrt{s_{N N}}=200 \mathrm{GeV} / \mathrm{c}$. At this energy, it is predicted by some models [2] that requiring a trigger during jet selection (leading hadron, minimum jet $p_{T}$, etc) will bias the jet population that fulfills these trigger requirements towards surface production. Jets produced in the center of the fireball have a high probability to be quenched such that they no longer fulfill the trigger requirement.

It may be possible to use this bias to systematically study the path length dependence of partonic energy loss by varying the trigger requirement, and thus the magnitude of the trigger bias. In these proceedings we present the first systematic attempt at this procedure, measuring the di-jet imbalance $A_{J}$ as a function of the jet definition in $\mathrm{Au}+\mathrm{Au}$, compared to a $\mathrm{p}+\mathrm{p}$ reference.

\section{Di-jet Imbalance Measurements at STAR}

STAR has published a measurement of the di-jet imbalance [3], which is defined as

$$
\left|A_{J}\right|=\frac{\left|p_{T}^{\text {lead }}-p_{T}^{\text {sublead }}\right|}{p_{T}^{\text {lead }}+p_{T}^{\text {sublead }}}
$$

where $p_{T}^{\text {lead }}$, and $p_{T}^{\text {sublead }}$ are the leading and subleading jet transverse momenta in central $(0-20 \%)$ $\mathrm{Au}+\mathrm{Au}$ collisions at $\sqrt{s_{N N}}=200 \mathrm{GeV}$. In this measurement, jets are selected using a "hard-core" trigger di-jet selection, by clustering only the hard constituents of the event: in this case, requiring $p_{T}>2.0 \mathrm{GeV} / c$, finding di-jets such that $p_{T}^{\text {lead }}>20.0 \mathrm{GeV} / c$ and $p_{T}^{\text {sublead }}>10.0 \mathrm{GeV} / c$, and requiring a calorimeter hit in the event with a transverse energy deposition $E_{T}>5.4 \mathrm{GeV} / c$. The hard-core selection has two effects: it eliminates the contribution from background fluctuations in $\mathrm{Au}+\mathrm{Au}$ events being tagged as jets, and also biases selection of true jets to the subset which has a relatively hard fragmentation pattern, with the majority of its energy found in highly energetic particles. If the event has a valid hard-core di-jet pair, then the event is reclustered using a much lower $\mathrm{p}_{\mathrm{T}}^{\text {const }}>0.2 \mathrm{GeV} / c$, and the resulting jets are matched to the hard-core jets such that $\Delta R=\sqrt{\Delta \phi^{2}+\Delta \eta^{2}}<R$, where $\Delta \eta=\eta^{\mathrm{HC}}-\eta^{\text {match }}$ and $\Delta \phi=\phi^{\mathrm{HC}}-\phi^{\text {match }}$. This allows the matched jet $\left|A_{J}\right|$ to be calculated and compared to those from $\mathrm{p}+\mathrm{p}$. The previous measurement found that, for $R=0.4$, the hard-core jets are significantly modified compared to $\mathrm{p}+\mathrm{p}$, whereas the matched jets are unmodified. This implies that any energy lost in the hard-core jets was found still within the initial jet cone, but in softer constituents. When the measurement was performed with $R=0.2$, the matched jets are also modified, which gives evidence of some intra-jet broadening between $R=0.2$ and $R=0.4$. 
In this new measurement we systematically vary the parameters of the jet-finding algorithm. We hold the jet $\mathrm{p}_{\mathrm{T}}$ cut constant for both the leading and subleading jet at 16.0 and $8.0 \mathrm{GeV} / \mathrm{c}$, respectively, and vary both the $p_{T}^{\text {const }}$ from $1.0 \mathrm{GeV} / c$ to $3.0 \mathrm{GeV} / c$ and the jet radius from 0.2 to 0.4 . We then use di-jet imbalance to measure the deviation of $\mathrm{Au}+\mathrm{Au}$ from embedded $\mathrm{p}+\mathrm{p}$ at each point in this two-dimensional jet definition space.

\section{Analysis Details}

The data shown were collected in $2007(\mathrm{Au}+\mathrm{Au})$ and $2006(\mathrm{p}+\mathrm{p})$ by the STAR detector at $\sqrt{s_{N N}}=200 \mathrm{GeV} / c$. Charged tracks are reconstructed by the time projection chamber (TPC) [4]. Tracks selected for this analysis are required to have a minimum of 20 fit points (out of a maximum of 46) in the TPC, a fraction of maximum possible fit points (based on event and track geometry) greater than 0.52 , have a DCA less than $1.0 \mathrm{~cm}$ to the primary vertex, and to have a pseudorapidity $|\eta|<1.0$. Neutral energy in the event is measured by the barrel electromagnetic calorimeter (BEMC) [5]. To avoid double counting energy from reconstructed charged tracks in the BEMC, the track helix is extrapolated to the calorimeter, and the $p_{T}$ of the track is subtracted from the matched tower transverse energy $\left(E_{T}\right)$. Tower energy is not allowed to become negative from this correction.

Events are selected by an online high tower (HT) trigger, requiring at least one BEMC tower signal with $E_{T}>5.4 \mathrm{GeV}$. They are also required to have a primary vertex position along the beam axis of $\left|v_{z}\right|<30 \mathrm{~cm}$. Event centrality is determined by the raw charged track multiplicity in the TPC within the pseudorapidity range $|\eta|<0.5$. In these proceedings, we only show results for $0-20 \%$ centrality.

Jets are reconstructed from charged tracks in the TPC and energy depositions in the BEMC using the anti- $k_{T}$ [6] algorithm in the FastJet package [7] with multiple radii, as described in the above section. The initial di-jet selection for the hard-core jets are clustered using all constituents above a variable $p_{T}$ as described in the previous section. It also requires $p_{T}^{\text {lead }}>16.0 \mathrm{GeV} / c$, $p_{T}^{\text {sublead }}>8.0 \mathrm{GeV} / c,\left|\eta^{\text {jet }}\right|<1.0-R$, and the leading and subleading jets must be back-to-back in the azimuthal angle, i.e. $\left|\phi^{\text {lead }}-\phi^{\text {sublead }}-\pi\right|<0.4$. Matched jets are always clustered with all constituents with $p_{T}>0.2 \mathrm{GeV} / c$, and geometrically matched to the hard-core jets, as described in Section 2. All jets' (hard-core and matched jets) momenta are corrected for background density using an area-based subtraction method giving $p_{T}^{\text {jet }}=p_{T}^{\text {measured }}-\rho^{\text {event }} A^{\text {jet }}$, where both the jet area $A^{\text {jet }}$ and the background density $\rho^{\text {event }}$ are calculated as described for the matched jets in STAR's previous measurement of $\left|A_{J}\right|$ [3].

For a meaningful comparison between $\mathrm{Au}+\mathrm{Au}$ and a $\mathrm{p}+\mathrm{p}$ reference, the effects of background fluctuations and detector inefficiencies must be taken into account. To achieve this, the $p+p$ data is embedded into minimum bias (MB) Au+Au data, in the same centrality range $(0-20 \%)$. During embedding, we account for the relative tracking efficiency $(90 \% \pm 7 \%)$ and relative tower energy scale $(100 \% \pm 2 \%)$. Systematic uncertainty is estimated on $\left|A_{J}\right|$ by varying the relative tracking efficiency and tower energy scale in the embedded $\mathrm{p}+\mathrm{p}$ by $1 \sigma$. 


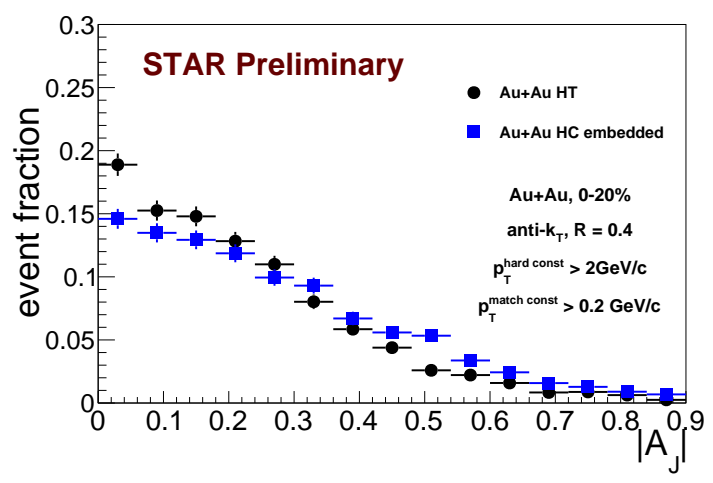

Figure 1: $\left|A_{J}\right|$ distributions for $\mathrm{Au}+\mathrm{Au}$ and $\mathrm{Au}+\mathrm{Au}$ hard-core jets embedded in min-bias $\mathrm{Au}+\mathrm{Au}$, with $p_{T}^{\text {const }}>2.0 \mathrm{GeV} / c$ and $R=0.4$.

\section{Results}

Fluctuations in the background energy density in the matched jets will lead to a smeared $\left|A_{J}\right|$ distribution. To estimate the sensitivity of the measurement to physical balancing due to correlated jet yield, as opposed to balancing due to background fluctuations, hard-core jets for each di-jet definition are embedded into minimum bias $\mathrm{Au}+\mathrm{Au}$ events of the same centrality. Therefore, there is no correlated jet-like yield below $2.0 \mathrm{GeV} / c$ in this embedded sample, and any balancing is purely due to the background. The $\left|A_{J}\right|$ is calculated for these embedded jets, and compared to the $\mathrm{Au}+\mathrm{Au}$ and embedded $\mathrm{p}+\mathrm{p}$ curves, as shown in Figure 1. To compare the similarity of two datasets (statistical errors only), we use a two sample Kolmogorov-Smirnov goodness-of-fit test (KS test) to extract a p-value. Using the KS test to quantify similarity between the embedded hard-core jets and the true $\mathrm{Au}+\mathrm{Au}$ matched jets, the result ( $p \ll 0.05$ for all di-jet definitions) gives quantitative evidence of sensitivity to physical balancing.
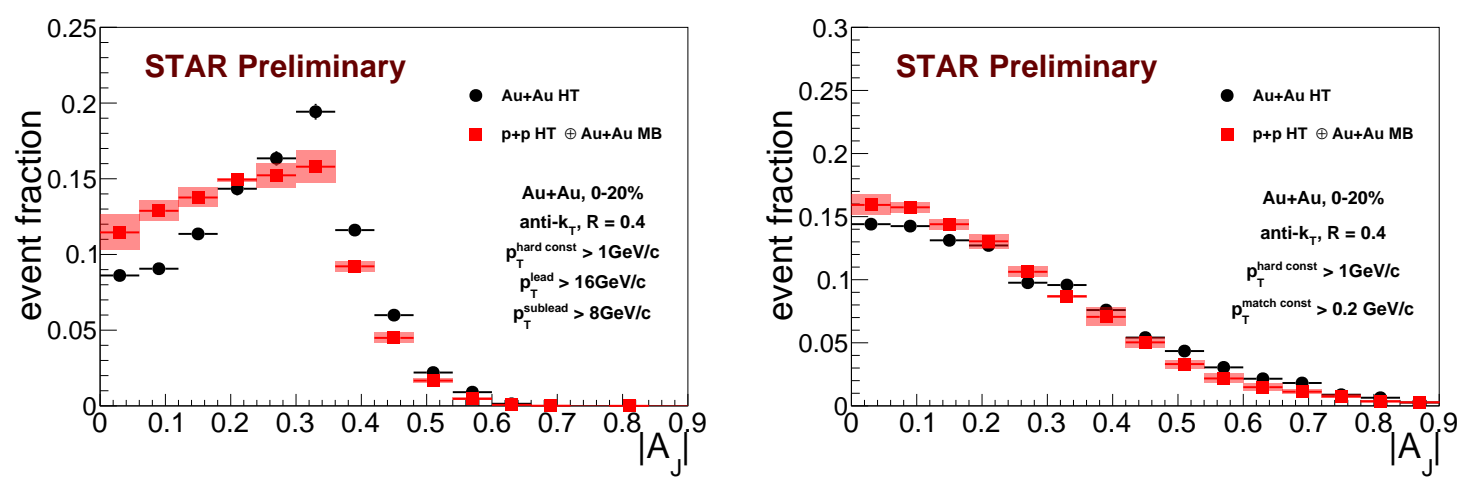

Figure 2: $\left|A_{J}\right|$ distributions for $\mathrm{Au}+\mathrm{Au}$ and embedded $\mathrm{p}+\mathrm{p}$, with $p_{T}^{\text {const }}>1.0 \mathrm{GeV} / c$ and $R=0.4$. Left: hard-core di-jet $\left|A_{J}\right|, \mathrm{KS}$ test p-value $\approx 0.0$. Right: matched di-jet $\left|A_{J}\right|, \mathrm{KS}$ test $\mathrm{p}$-value $=10^{-16}$.

We calculate $\left|A_{J}\right|$ for hard-core jets in $\mathrm{Au}+\mathrm{Au}$ and embedded $\mathrm{p}+\mathrm{p}$, varying the minimum constituent $p_{T}^{\text {const }}$ between 1.0 and $3.0 \mathrm{GeV} / c$ in steps of 0.5 , and varying the jet-finder radius $R$ from 


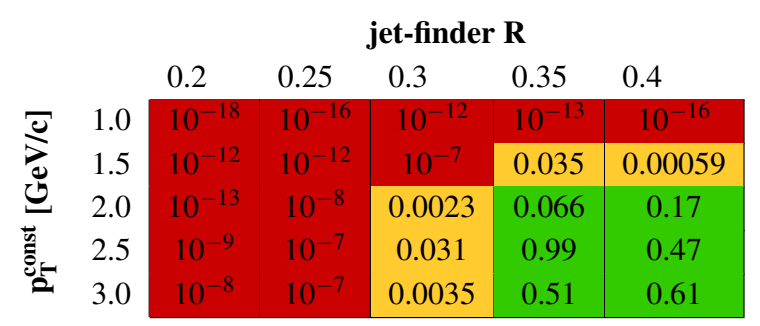

\begin{tabular}{|c|c|c|c|c|c|}
\hline & \multicolumn{5}{|c|}{ jet-finder $\mathbf{R}$} \\
\hline & 0.2 & 0.25 & 0.3 & 0.35 & 0.4 \\
\hline 1.0 & $10^{-}$ & $10^{-}$ & 0.0010 & 0.11 & 0.33 \\
\hline 1.5 & $10^{-16}$ & $10^{-6}$ & 0.0028 & 0.086 & 0.90 \\
\hline 2.0 & $10^{-18}$ & $10^{-7}$ & 0.0014 & 0.017 & 0.63 \\
\hline 2.5 & $10^{-13}$ & $10^{-6}$ & 0.0057 & 0.12 & 0.62 \\
\hline 3.0 & $10^{-10}$ & $10^{-7}$ & 0.027 & 0.23 & 0.55 \\
\hline
\end{tabular}

Table 1: Kolmogorov-Smirnov test p-values for matched di-jet $\left|A_{J}\right|$ comparison between Au+Au and embedded $\mathrm{p}+\mathrm{p}$. colors: red, imbalanced $(p<0.0005)$; yellow, semi-balanced $(0.0005<p<0.05)$; green, balanced $(p>0.05)$. Left: hard-core and matched jet radius both varied. Increased balance at greater $p_{T}^{\text {const }}$ and R. Right: hard-core radius fixed at $R=0.2$, matched jet radius varied. Balance independent of $p_{T}^{\text {const }}$.

0.2 to 0.4 in steps of 0.05 , creating 25 di-jet definitions in total. An example is shown on the left side of Figure 2. For all $\left(p_{T}^{\text {const }}, R\right)$ pairs, the p-value is significantly less than $1 \%$, from which we infer the two datasets did not come from the same underlying probability distribution. Because this is true for all $\left(p_{T}^{\text {const }}, R\right)$ pairs, we can say that the hard-core jets in $\mathrm{Au}+\mathrm{Au}$ are always modified with respect to $\mathrm{p}+\mathrm{p}$ in the phase space explored in this analysis. We then perform our matching procedure; for each found hard-core di-jet pair, we cluster the event again using $p_{T}^{\text {const }}>0.2 \mathrm{GeV} / c$ and the same radius as the hard-core jets, and match the two sets of jets geometrically, as described above; an example $\left|A_{J}\right|$ distribution is shown on the right side of Figure 2. When we calculate the KS-test p-value for the matched jets, we see a smooth transition from statistically different distributions at low $R$ and low $p_{T}$ to statistically similar distributions at larger $R$ and larger $p_{T}$, shown in Table 1. This shows the radial dependence of the energy loss, and the evolution of energy loss as a function of the hard-core $p_{T}$ cut.

Finally, we explore the radial dependence of the quenched energy by scanning the matched jet radius for a fixed hard-core radius. This time, starting from a fixed hard-core jet radius of 0.2 with varying $p_{T}^{\text {const }}$, we match to jets of varying radii, from 0.2 to 0.4 . Using the KS test again, the balance is restored at $R=0.35$, independent of the hard-core $p_{T}^{\text {const }}$, as shown in Table 1 .

\section{Summary}

We have demonstrated that, by modifying the di-jet definition used during jet-finding, we can select jets that are relatively more or less modified compared to a $p+p$ reference. This opens up the possibility of jet geometry engineering, and may help constrain the path length dependence of partonic energy loss in the QGP at RHIC. Further analysis with increased statistics is planned to include other important dimensions, such as centrality and jet $\mathrm{p}_{\mathrm{T}}$ scale.

\section{References}

[1] B. I. Abelev et al., Phys. Rev. Lett. 97, 252001 (2006)

[2] T. Renk, Phys. Rev. C 88, no. 5, 054902 (2013)

[3] L. Adamczyk, et al., Phys. Rev. Lett. 119, no. 6, 062301 (2017)

[4] M. Anderson, et al., Nucl. Instrum. Meth. A 499, 659 (2003) 
[5] M. Beddo, et al., Nucl. Instrum. Meth. A 499, 725 (2003)

[6] M. Cacciari, et al., JHEP 0804, 063 (2008)

[7] M. Cacciari, et al., Eur. Phys. J. C 72, 1896 (2012) 